\title{
UNIT-REGULARITY OF REGULAR NILPOTENT ELEMENTS
}

\author{
DINESH KHURANA
}

\begin{abstract}
Let $a$ be a regular element of a ring $R$. If either $K:=\mathrm{r}_{\mathrm{R}}(\mathrm{a})$ has the exchange property or every power of $a$ is regular, then we prove that for every positive integer $n$ there exist decompositions

$$
R_{R}=K \oplus X_{n} \oplus Y_{n}=E_{n} \oplus X_{n} \oplus a Y_{n},
$$

where $Y_{n} \subseteq a^{n} R$ and $E_{n} \cong R / a R$. As applications we get easier proofs of the results that a strongly $\pi$-regular ring has stable range one and also that a strongly $\pi$-regular element whose every power is regular is unit-regular.
\end{abstract}

An element $a$ of a ring $R$ is called strongly $\pi$-regular if both chains $a R \supseteq a^{2} R \supseteq a^{3} R \ldots$ and $R a \supseteq$ $R a^{2} \supseteq R a^{3} \ldots$ stabilize. If every element of $R$ is strongly $\pi$-regular, then $R$ is called a strongly $\pi$-regular ring. In [1] Pere Ara proved a wonderful result that a strongly $\pi$-regular ring has stable range one. Ara's proof is on the following lines. As a strongly $\pi$-regular ring is an exchange ring and an exchange ring has stable range one if and only if every regular element is unit-regular, it is enough to show that every regular element of a strongly $\pi$-regular ring is unit-regular. Suppose $a$ is a regular element of a strongly $\pi$-regular ring. By [7, Proposition 1] there exist $n \in \mathbb{N}$, an idempotent $e$ and a unit $u$ in $R$ with $a^{n}=e u$ such that $a, e$ and $u$ commute with each other. Then $e a$ is a unit in $e$ Re with inverse $e a^{n-1} u^{-1}$ and $(1-e) a$ is a regular nilpotent element of the exchange ring $(1-e) R(1-e)$. As $a=e a+(1-e) a$ and $e a$ is unit-regular in $e R e$, we will get that $a$ is unit-regular if we can show that $(1-e) a$ is unit-regular in $(1-e) R(1-e)$. So the result will follow if we can show that a regular nilpotent element of an exchange ring is unit-regular. This is the crucial result proved by Ara in [1] and an easier proof of this will follow from our Theorem 2.

In [5, Theorem 5.8] Goodearl and Menal proved that a regular strongly $\pi$-regular ring is unit-regular. The proof of [5. Theorem 5.8] can be adapted to prove that if $a$ is a strongly $\pi$-regular element of any $\operatorname{ring} R$ such that $a^{n}$ is regular for every $n \in \mathbb{N}$, then $a$ is unit-regular. A different proof of this result was given by Beidar, O'Meara and Raphael in [3, Corollary 3.7]. Suppose $a$ is a strongly $\pi$-regular element of a ring $R$ whose each power is regular. Then as above there exist $n \in \mathbb{N}$, an idempotent $e$ and a unit $u$ in $R$ with $a^{n}=e u$ such that $a, e$ and $u$ commute with each other. As seen above it will follow that $a$ is unit-regular if we can prove that $(1-e) a$ is unit-regular in $(1-e) R(1-e)$. As $(1-e) a$ is nilpotent and its each power is regular in $(1-e) R(1-e)$, it is enough to prove that a nilpotent element whose each power is regular is unit-regular. An easier proof of this will follow from our Theorem 4.

Recently Ara and O'Meara in [2] and Pace and Šter in [8] have shown that a regular nilpotent element in general may not be unit-regular.

By $A \subseteq{ }^{\oplus} B$ we shall mean that $A$ is a summand of the module $B$. We will tacitly use the fact that a regular element $a \in R$ is unit-regular if and only if $\mathrm{r}_{\mathrm{R}}(\mathrm{a}) \cong \mathrm{R} / \mathrm{aR}$, where $\mathrm{r}_{\mathrm{R}}(\mathrm{a})=\{\mathrm{x} \in \mathrm{R}: \mathrm{ax}=0\}$.

Lemma 1 [4, Corollary 3.9]. If $M$ has the exchange property and $A=M \oplus B \oplus C=\bigoplus_{I} A_{i} \oplus C$, then there exists a decomposition $A_{i}=D_{i} \oplus E_{i}$ of each $A_{i}$ such that $A=M \oplus \bigoplus_{I} D_{i} \oplus C$.

2010 Mathematics Subject Classification. Primary 16E50, Secondary $19 \mathrm{~B} 10$.

Key words and phrases. Regular elements, nilpotent elements, strongly $\pi$ - regular elements, stable range one. 
Theorem 2. Let a be a regular element of a ring $R$ such that the right $R$-module $K:=\mathrm{r}_{\mathrm{R}}(\mathrm{a})$ has the exchange property. Then for every $n \in \mathbb{N}$ there exist decompositions

$$
R_{R}=K \oplus X_{n} \oplus Y_{n}=E_{n} \oplus X_{n} \oplus a Y_{n},
$$

where $Y_{n} \subseteq a^{n} R$ and $E_{n} \cong R / a R$. If $a^{n}=0$, then $a Y_{n}=Y_{n}=0$ and so $K \cong E_{n} \cong R / a R$ implying that a is unit-regular.

Proof. For every positive integer $i$ we will inductively construct right ideals $A_{i}, A_{i}^{\prime}, Y_{i}$ of $R$ such that for every $j \geq 1$,

$$
R=K \oplus\left(\bigoplus_{i=1}^{j} A_{i}\right) \oplus Y_{j}=\left(\bigoplus_{i=1}^{j} A_{i}\right) \oplus\left(A_{j}^{\prime} \oplus a A_{j}\right) \oplus a Y_{j},
$$

where $Y_{j} \subseteq a^{j} R$ and $A_{j}^{\prime} \oplus a A_{j}=A_{j+1} \oplus A_{j+1}^{\prime} \cong R / a R$. Then we have the desired decompositions by putting $X_{n}=\bigoplus_{i=1}^{n} A_{i}$ and $E_{n}=A_{n}^{\prime} \oplus a A_{n}$.

We have $R=K \oplus B=A \oplus a R$ for some right ideals $A$ and $B$ of $R$. As $K_{R}$ has the exchange property, by Lemma 1 we have decompositions $A=A_{1} \oplus A_{1}^{\prime}$ and $a R=Y_{1} \oplus Y_{1}^{\prime}$ such that $R=K \oplus A_{1} \oplus Y_{1}$. As $K \cap\left(A_{1} \oplus Y_{1}\right)=0, a R=a A_{1} \oplus a Y_{1}$ and $a A_{1} \cong A_{1}$. So $R=A_{1} \oplus A_{1}^{\prime} \oplus a R=A_{1} \oplus A_{1}^{\prime} \oplus a A_{1} \oplus a Y_{1}$ implying that

$$
R=K \oplus A_{1} \oplus Y_{1}=A_{1} \oplus\left(A_{1}^{\prime} \oplus a A_{1}\right) \oplus a Y_{1},
$$

where $Y_{1} \subseteq a R$ and $A_{1}^{\prime} \oplus a A_{1} \cong A_{1}^{\prime} \oplus A_{1}=A \cong R / a R$.

Now suppose we have found the right ideals $A_{i}, A_{i}^{\prime}, Y_{i}$ for $i=1, \ldots, n$ such that $(*)$ holds for every $j=1, \ldots, n$ with $Y_{i} \subseteq a^{i} R, A_{i}^{\prime} \oplus a A_{i} \cong R / a R$ for every $i=1, \ldots, n$ and $A_{i}^{\prime} \oplus a A_{i}=A_{i+1}^{\prime} \oplus A_{i+1}$ for every $i=1, \ldots, n-1$. As $K$ has the exchange property and

$$
R=K \oplus\left(\bigoplus_{i=1}^{n} A_{i}\right) \oplus Y_{n}=\left(\bigoplus_{i=1}^{n} A_{i}\right) \oplus\left(A_{n}^{\prime} \oplus a A_{n}\right) \oplus a Y_{n},
$$

by Lemma 1 we have decompositions $A_{n}^{\prime} \oplus a A_{n}=A_{n+1} \oplus A_{n+1}^{\prime}$ and $a Y_{n}=Y_{n+1} \oplus Y_{n+1}^{\prime}$ such that

$$
R=K \oplus\left(\bigoplus_{i=1}^{n+1} A_{i}\right) \oplus Y_{n+1}
$$

So $a R=\bigoplus_{i=1}^{n+1} a A_{i} \oplus a Y_{n+1}$ and $a A_{n+1} \cong A_{n+1}$. Now as $A_{j}^{\prime} \oplus a A_{j}=A_{j+1} \oplus A_{j+1}^{\prime}$ for every $j=1, \ldots, n$ we have

$$
R=A_{1} \oplus A_{1}^{\prime} \oplus a R=A_{1} \oplus A_{1}^{\prime} \oplus \bigoplus_{i=1}^{n+1} a A_{i} \oplus a Y_{n+1}=\left(\bigoplus_{i=1}^{n+1} A_{i}\right) \oplus\left(A_{n+1}^{\prime} \oplus a A_{n+1}\right) \oplus a Y_{n+1},
$$

with $Y_{n+1} \subseteq a^{n+1} R$ and $A_{n+1}^{\prime} \oplus a A_{n+1} \cong A_{n+1}^{\prime} \oplus A_{n+1}=A_{n}^{\prime} \oplus a A_{n} \cong R / a R$.

Lemma 3 [6, Lemma 2.8]. Let $P_{R}$ be a projective module and $P=A+B$ where $A \subseteq{ }^{\oplus} P$. Then $B=C \oplus D$ for some submodules $C$ and $D$ such that $P=A \oplus C$.

Theorem 4. Let a be an element of a ring $R$ such that $a^{n}$ is regular for every positive integer $n$. Then for every $n \in \mathbb{N}$ there exist decompositions

$$
R_{R}=K \oplus X_{n} \oplus Y_{n}=E_{n} \oplus X_{n} \oplus a Y_{n},
$$

where $K=\mathrm{r}_{\mathrm{R}}(\mathrm{a}), Y_{n} \subseteq a^{n} R, K \oplus Y_{n}=K+a^{n} R$ and $E_{n} \cong R / a R$. If $a^{n}=0$, then $K \cong E_{n} \cong R / a R$ implying that a is unit-regular. 
Proof. For every positive integer $i$ we will inductively construct right ideals $A_{i}, A_{i}^{\prime}, Y_{i}$ of $R$ such that for every $j \geq 1$,

$$
R=K \oplus\left(\bigoplus_{i=1}^{j} A_{i}\right) \oplus Y_{j}=\left(\bigoplus_{i=1}^{j} A_{i}\right) \oplus\left(A_{j}^{\prime} \oplus a A_{j}\right) \oplus a Y_{j},
$$

where $Y_{j} \subseteq a^{j} R, a Y_{j}=a^{j+1} R$ and $A_{j}^{\prime} \oplus a A_{j}=A_{j+1} \oplus A_{j+1}^{\prime} \cong R / a R$. Then we have the desired decompositions by putting $X_{n}=\bigoplus_{i=1}^{n} A_{i}$ and $E_{n}=A_{n}^{\prime} \oplus a A_{n}$.

Note that the left multiplication by $a$ induces an epimorphism from $R \rightarrow a R / a^{n+1} R$ with kernel $K+a^{n} R$. As $a$ and $a^{n+1}$ are regular, $a R / a^{n+1} R$ is projective implying that $K+a^{n} R \subseteq{ }^{\oplus} R_{R}$ for each $n$. By Lemma $3, K+a R=K \oplus Y_{1}$ for some $Y_{1} \subseteq a R$. If $Y_{1}^{\prime}=a R \cap K$, then on intersecting with $a R$ we have $a R=Y_{1}^{\prime} \oplus Y_{1}, a^{2} R=a Y_{1}$ and $Y_{1}^{\prime} \subseteq{ }^{\oplus} R_{R}$. So $K=Y_{1}^{\prime} \oplus A_{1}^{\prime}$ for some $A_{1}^{\prime}$. Also for some right ideal $A_{1}$ we have $R=(K+a R) \oplus A_{1}=K \oplus Y_{1} \oplus A_{1}=Y_{1}^{\prime} \oplus A_{1}^{\prime} \oplus Y_{1} \oplus A_{1}$. As $K \cap\left(A_{1} \oplus Y_{1}\right)=0$, $a R=a A_{1} \oplus a Y_{1}$ and $a A_{1} \cong A_{1}$. So $R=A_{1} \oplus A_{1}^{\prime} \oplus a R=A_{1} \oplus A_{1}^{\prime} \oplus a A_{1} \oplus a Y_{1}$. Thus

$$
R=K \oplus A_{1} \oplus Y_{1}=A_{1} \oplus\left(A_{1}^{\prime} \oplus a A_{1}\right) \oplus a Y_{1},
$$

where $Y_{1} \subseteq a R, K \oplus Y_{1}=K+a R$ and $A_{1}^{\prime} \oplus a A_{1} \cong A_{1}^{\prime} \oplus A_{1} \cong R / a R$.

Now suppose we have found the right ideals $A_{i}, A_{i}^{\prime}, Y_{i}$ for $i=1, \ldots, n$ such that we have decompositions as in (**) for every $j=1, \ldots, n$ with $Y_{i} \subseteq a^{i} R, K \oplus Y_{i}=K+a^{i} R, A_{i}^{\prime} \oplus a A_{i} \cong R / a R$ for every $i=1, \ldots, n$ and $A_{i}^{\prime} \oplus a A_{i}=A_{i+1}^{\prime} \oplus A_{i+1}$ for every $i=1, \ldots, n-1$. By Lemma $3, K+a^{n+1} R=K \oplus Y_{n+1}$ for some $Y_{n+1} \subseteq a^{n+1} R$. Note that $a Y_{n}=a^{n+1} R \subseteq K \oplus Y_{n+1}$ and $\left(K \oplus Y_{n+1}\right) \cap \bigoplus_{i=1}^{n} A_{i} \subseteq$ $\left(K \oplus Y_{n}\right) \cap \bigoplus_{i=1}^{n} A_{i}=0$. As $K+a^{n} R \subseteq^{\oplus} R_{R}$ for each $n, K \oplus Y_{n+1}=K+a^{n+1} R \subseteq{ }^{\oplus} K+a^{n} R=K \oplus Y_{n}$ and $K \oplus Y_{n+1} \oplus \bigoplus_{i=1}^{n} A_{i} \subseteq{ }^{\oplus} K \oplus Y_{n} \oplus \bigoplus_{i=1}^{n} A_{i}=R$. Thus

$R=\left(K \oplus Y_{n+1}\right)+R=\left(K \oplus Y_{n+1}\right)+\left(\bigoplus_{i=1}^{n} A_{i} \oplus\left(A_{n}^{\prime} \oplus a A_{n}\right) \oplus a Y_{n}\right)=\left(K \oplus Y_{n+1} \oplus \bigoplus_{i=1}^{n} A_{i}\right)+\left(A_{n}^{\prime} \oplus a A_{n}\right)$.

Again by Lemma $3, A_{n}^{\prime} \oplus a A_{n}=A_{n+1} \oplus A_{n+1}^{\prime}$ such that $R=K \oplus Y_{n+1} \oplus \bigoplus_{i=1}^{n+1} A_{i}$. So $a R=$ $\bigoplus_{i=1}^{n+1} a A_{i} \oplus a Y_{n+1}, a A_{n+1} \cong A_{n+1}$. Now as $A_{j}^{\prime} \oplus a A_{j}=A_{j+1} \oplus A_{j+1}^{\prime}$ for every $j=1, \ldots, n$ we have

$$
R=A_{1} \oplus A_{1}^{\prime} \oplus a R=A_{1} \oplus A_{1}^{\prime} \oplus \bigoplus_{i=1}^{n+1} a A_{i} \oplus a Y_{n+1}=\left(\bigoplus_{i=1}^{n+1} A_{i}\right) \oplus\left(A_{n+1}^{\prime} \oplus a A_{n+1}\right) \oplus a Y_{n+1},
$$

with $Y_{n+1} \subseteq a^{n+1} R, K \oplus Y_{n+1}=K+a^{n+1} R$ and $A_{n+1}^{\prime} \oplus a A_{n+1} \cong A_{n+1}^{\prime} \oplus A_{n+1}=A_{n}^{\prime} \oplus a A_{n} \cong R / a R$.

Acknowledgements: We thank Pace P. Nielsen for useful comments that improved the presentation. We also thank the referee for many corrections and improvements.

\section{REFERENCES}

1. Pere Ara, Strongly $\pi$-regular rings have stable range one, Proc. Amer. Math. Soc. 124 (1996), 3293-3298.

2. Pere Ara and K. C. O'Meara, The nilpotent regular element problem, arXiv: 1509.08862 (2015), $10 \mathrm{pp}$.

3. K. I. Beidar, K. C. O'Meara, and R. M. Raphael, On uniform diagonalization of matrices over regular rings and one-accessible regular algebras, Comm. Alg. 32 (2004), 3543-3562.

4. P. Crawley and B. Jónsson, Refinements for infinite direct decompositions of algebraic systems, Pacific J. Math. 14 (1964), 797-855.

5. K. R. Goodearl and P. Menal, Stable range one for rings with many units, J. Pure Appl. Alg. 54 (1988), $261-287$.

6. W. K. Nicholson, Lifting idempotents and exchange rings, Trans. Amer. Math. Soc. 229 (1977), 269-278.

7. _ Strongly clean rings and fitting's lemma, Comm. Alg. 27 (1999), 3583-3592.

8. Pace P. Nielsen and Janez Šter, Connections between unit-regularity, regularity, cleanness, and strong cleanness of elements and rings, arXiv: 1510.03305 (2015), 17 pp. 
Department of Mathematics, Panjab University, Chandigarh-160014, India

E-mail address: dkhurana@pu.ac.in 